\title{
On the best quantity reconstructions for a well balanced finite volume method used to solve the shallow water wave equations with a wet/dry interface
}

\author{
Sudi Mungkasi ${ }^{1} \quad$ Stephen G. Roberts ${ }^{2}$
}

(Received 29 November 2009; revised 28 February 2010)

\begin{abstract}
Well balanced finite volume methods used to solve the shallow water wave equations are designed to preserve the steady state of a 'lake at rest'. Unfortunately, for problems involving wet/dry interfaces, this steady state is not preserved unless the involved quantities are reconstructed with care. We test four reconstruction options: stage and momentum (where bed is fixed); stage and velocity (where bed is fixed); stage, water height, and velocity; and stage, bed, and velocity (with modification at wet/dry interfaces). Reconstructions based on stage, water height, and velocity are shown to preserve the steady state problem and accurately solve a representative unsteady state problem, whereas the other options lead to problems in both maintaining the steady solution and accurately solving non-steady problems. Our results indicate the appropriate choice of reconstruction variables for
\end{abstract}

http://anziamj . austms.org.au/ojs/index.php/ANZIAMJ/article/view/2576 gives this article, (c) Austral. Mathematical Soc. 2010. Published March 3, 2010. ISSN 1446-8735. (Print two pages per sheet of paper.) 
various situations when solving the shallow water wave equations using finite volume methods.

\section{Contents}

1 Introduction

2 Well balanced methods and schemes

3 Quantity reconstructions

C52

4 Numerical tests

C55

4.1 Steady state: a lake at rest . . . . . . . . . . . . C56

4.2 Unsteady state: oscillation on a parabolic bed . . . . . . .

C59

5 Conclusions

C60

References

C64

\section{Introduction}

The conservative form of the one dimensional shallow water wave equations is

$$
\mathbf{q}_{\mathbf{t}}+\mathbf{f}(\mathbf{q})_{\mathrm{x}}=\mathbf{s},
$$

in which quantity vector $\mathbf{q}$, the flux function $\mathbf{f}$, and the source term $\mathbf{s}$ are

$$
\mathbf{q}=\left[\begin{array}{c}
\mathrm{h} \\
\mathrm{hu}
\end{array}\right], \quad \mathbf{f}=\left[\begin{array}{c}
\mathrm{hu} \\
h^{2} \mathrm{u}+\frac{1}{2} g h^{2}
\end{array}\right], \quad \text { and } \quad \mathbf{s}=\left[\begin{array}{c}
0 \\
-g h z_{x}
\end{array}\right] .
$$

Here, $x$ represents the one dimensional spatial variable, $t$ represents the time variable, $\mathfrak{u}=\mathfrak{u}(x, t)$ denotes the water velocity, $h=h(x, t)$ denotes the 
water height (depth), $z=z(x)$ denotes the topography (bed), $w=h+z$ is the water stage, and $\mathrm{g}$ is the constant of gravitation.

A finite volume method for solving these shallow water wave equations is essentially defined in terms of the reconstruction of flow quantities at the interfaces between cells, and the subsequent calculation of fluxes across these interfaces.

A robust and accurate finite volume method should be able to deal with both steady state problems and unsteady state problems, and in particular should be able to deal with wet/dry interfaces. We consider two representative problems with wet/dry interfaces, namely: a steady 'lake at rest with shoreline' and an unsteady 'oscillating lake on a parabolic bed'. The first problem has a fixed wet/dry interface, while the second problem has a moving wet/dry interface. We investigate the use of various reconstruction strategies when solving these two problems, and make recommendations on an appropriate choice reconstruction which leads to an accurate and robust well balanced method.

\section{Well balanced methods and schemes}

An interesting problem arises for the 'simple' lake at rest problem. In this case the vertical pressure term $\left(\frac{1}{2} g h^{2}\right)_{x}$ needs to precisely balance the bed gravity term $-g h z_{x}$. For a numerical scheme, the discretization of these two terms needs to be carefully undertaken to maintain this balance. A finite volume method is said to be well balanced if it preserves this balance; that is, if it preserves the lake at rest solution $(u=0, w=$ constant $)$. The importance of well balanced schemes also extends to problems which are small perturbations of the lake at rest problem, such as tsunamis in the deep ocean.

To define a finite volume method we need to specify the way flow quantities are reconstructed at the interfaces between cells. 
Suppose at the $\left(i+\frac{1}{2}\right)$ th interface between the $i$ th and $(i+1)$ th cells, we have used a standard method to reconstruct the values of water depth and bed, $h_{i, r}$ and $z_{i, r}$ on the right edge of the ith cell and $h_{i+1, l}$ and $z_{i+1, l}$ on the left edge of the $(i+1)$ th cell.

To obtain a well balanced scheme, we use the hydrostatic reconstruction originally proposed by Audusse et al. [1] and extended by Noelle et al. [3]. The new reconstructed values of $h$ and $z$ at the $\left(i+\frac{1}{2}\right)$ th interface are

$$
\begin{aligned}
z_{i+\frac{1}{2}}^{*} & :=\max \left\{z_{i, r}, z_{i+1, l}\right\}, \\
h_{i, r}^{*} & :=\max \left\{0, h_{i, r}+z_{i, r}-z_{i+\frac{1}{2}}^{*}\right\}, \\
h_{i+1, l}^{*} & :=\max \left\{0, h_{i+1, l}+z_{i+1, l}-z_{i+\frac{1}{2}}^{*}\right\} .
\end{aligned}
$$

The values for $\mathrm{h}^{*}$ lead to auxiliary values for the conserved quantities, $\mathbf{Q}^{*}=$ $\left(h^{*}, h^{*} u\right)^{\top}$.

A semi-discrete version of our well balanced finite volume scheme is

$$
\Delta x_{i} \frac{d}{d t} Q_{i}+\mathcal{F}^{r}\left(Q_{i}, Q_{i+1}, z_{i, r}, z_{i+1, l}\right)-\mathcal{F}^{l}\left(Q_{i-1}, Q_{i}, z_{i-1, r}, z_{i, l}\right)=S_{i}^{(j)},
$$

where the right and left numerical fluxes of the ith cell are respectively calculated at $x_{i+1 / 2}$ and $x_{i-1 / 2}$, and

$$
\mathcal{F}^{\mathrm{r}}\left(\mathbf{Q}_{\mathrm{i}}, \mathbf{Q}_{\mathrm{i}+1}, z_{\mathrm{i}, \mathrm{r}}, z_{\mathrm{i}+1, \mathrm{l}}\right):=\mathbf{F}\left(\mathbf{Q}_{\mathrm{i}, \mathrm{r}}^{*}, \mathbf{Q}_{i+1, \mathrm{l}}^{*}\right)+\mathbf{S}_{\mathrm{i}, \mathrm{r}},
$$

and

$$
\mathcal{F}^{\mathrm{l}}\left(\mathbf{Q}_{i-1}, \mathbf{Q}_{i}, z_{i-1, l}, z_{i, r}\right):=\mathbf{F}\left(\mathbf{Q}_{i-1, r}^{*}, \mathbf{Q}_{i, l}^{*}\right)+\mathbf{S}_{i, l} .
$$

Here, $\mathbf{Q}$ is the approximation of the vector $\mathbf{q}$, and $\mathbf{F}$ is a conservative numerical flux consistent with the homogeneous shallow water wave equations computed in such a way that the method is stable. In addition,

$$
S_{i, r}:=\left[\begin{array}{c}
0 \\
\frac{g}{2} h_{i, r}^{2}-\frac{g}{2}\left(h_{i, r}^{*}\right)^{2}
\end{array}\right], \quad S_{i, l}:=\left[\begin{array}{c}
0 \\
\frac{g}{2} h_{i, l}^{2}-\frac{g}{2}\left(h_{i, l}^{*}\right)^{2}
\end{array}\right]
$$




\begin{tabular}{c|l} 
TABLE 1: Four combinations of quantity reconstructions \\
\hline \hline Combination & Reconstructed quantities \\
\hline A & $\begin{array}{l}\text { stage } w, \text { and momentum } p \\
\text { (where bed } z \text { is fixed) }\end{array}$ \\
\hline B & $\begin{array}{l}\text { stage } w, \text { and velocity } u \\
\text { (where bed } z \text { is fixed) }\end{array}$ \\
\hline C & stage $w$, water height $h$, and velocity $u$ \\
\hline D & stage $w$, bed $z$, and velocity $u$ \\
\hline
\end{tabular}

are the corrections due to the water height modification in the hydrostatic reconstruction. Furthermore, the index $\mathbf{j}$ of $\mathbf{S}_{\mathfrak{i}}^{(\mathbf{j})}$ in equation (6) denotes the order of the numerical source term. First and second order numerical source terms are

$$
\mathbf{S}_{i}^{(1)}:=\left[\begin{array}{l}
0 \\
0
\end{array}\right] \quad \text { and } \quad \mathbf{S}_{i}^{(2)}:=\left[\begin{array}{c}
0 \\
g \frac{h_{i, l}+h_{i, r}}{2}\left(z_{i, l}-z_{i, r}\right)
\end{array}\right] .
$$

\section{Quantity reconstructions}

For the one dimensional shallow water wave equations, we consider five quantities: water height $h$, bed $z$, stage $w$, water velocity $u$, and momentum $p=h u$. Reconstructing two elements of the set $\{h, z, w\}$ together with one element of the set $\{\boldsymbol{u}, \boldsymbol{p}\}$ is enough to solve the equations in general. We are interested in those reconstructions which involve the reconstruction of stage $w$ as it is this quantity which is most important in the development of a well balanced method.

Four combinations of reconstruction quantities, as shown in Table 1, are considered. Note that they are not the only combinations available. Combination $\mathrm{C}$ was used by Audusse et al. [1], and we test the performance of this combination against the other combinations. Combination A, combination $\mathrm{B}$, and combination $\mathrm{C}$ never result in negative water height, while 
combination $\mathrm{D}$ results in negative water height at the wet/dry interface if it is applied naïvely. In addition, combination A and combination B allow piece-wise continuous linear approximation of the bed, while combination $\mathrm{C}$ and combination $\mathrm{D}$ lead to piece-wise discontinuous linear approximation of the bed.

When a second order method is used, combination D leads to negative water height if a modification is not made at the wet/dry interface [1]. In order to get a well balanced method that is physically valid, negative water height is not allowed. Therefore, we modify the quantity reconstructions around the wet/dry interfaces as follows. Consider the steady state of a lake at rest. We use the minmod limiter. Suppose that a wet/dry interface occurs at the ith cell and that $z_{i, r}>w_{i, r}$. Then we define an auxiliary variable $\delta=z_{i, r}-w_{i, r}$. So as to maintain conservation, we modify the value of $z$ on both the right and on the left of the ith cell

$$
z_{i, r}^{\text {new }}=w_{i, r} \quad \text { and } \quad z_{i, l}^{\text {new }}=z_{i, l}+\delta .
$$

These new values replace the old ones and are used in subsequent computations. Such a modification is shown in Figure 1. This modification forces the water height reconstruction to be nonnegative, so that a method using combination D can be used to solve steady state problems. Unfortunately, a method using combination $\mathrm{D}$ with this modification leads to unphysical solutions at wet/dry areas when it is used to solve unsteady state problems, as we will see in Subsection 4.2.

Throughout Section 4, the well balanced scheme using combination A of reconstruction quantities is called Method A; likewise, Method B, Method C, and Method D denote the well balanced schemes using combination B, combination $\mathrm{C}$, and combination $\mathrm{D}$ respectively. Note that we apply the modification at the wet/dry interface for Method D. 


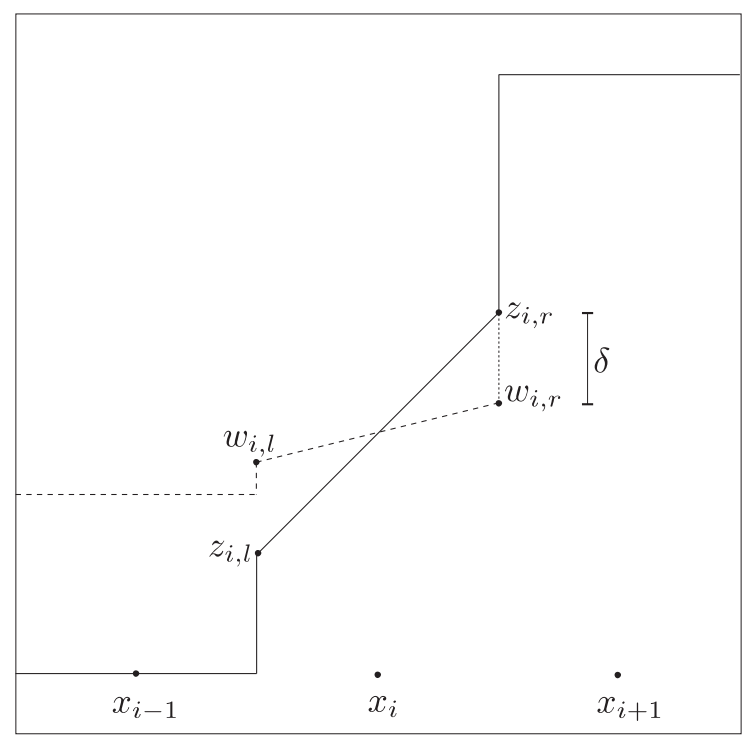

(a) Negative water height occurs

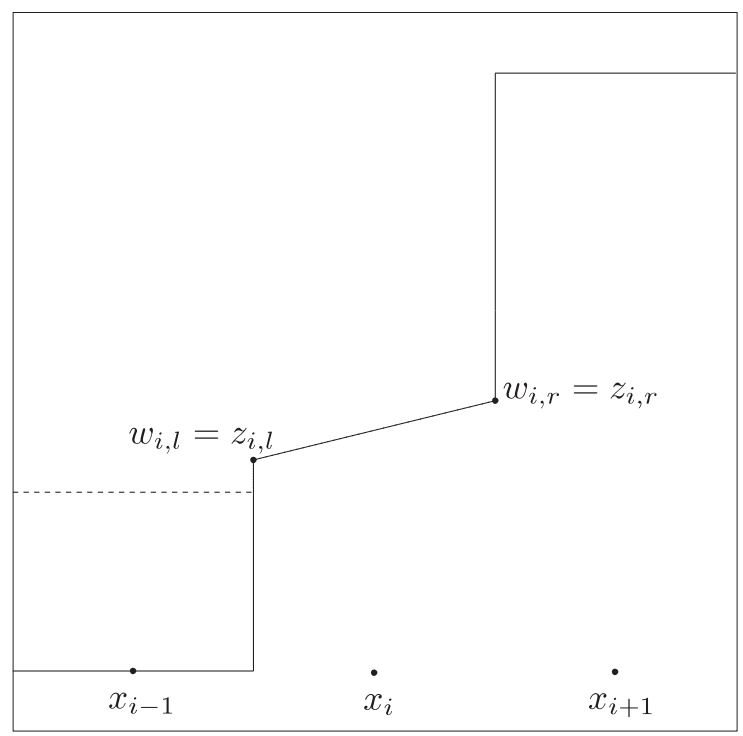

(b) Modified reconstruction

Figure 1: Modification at wet/dry interface for combination D, using minmod limiter. Free surface (dashed line). Water bed (continuous line). 


\section{$4 \quad$ Numerical tests}

In all of the simulations in this article, we use the second order source term $\mathbf{S}_{i}^{(2)}$ and second order spatial and temporal discretization. Note that first order discretization is not investigated as it leads to excessively diffusive solutions.

We use the central upwind formulation proposed by Kurganov, Noelle and Petrova [2] to compute the numerical fluxes. This method is efficient; it does not require a Riemann solver or a characteristic decomposition.

Quantities are measured in the sI system, and the constant of gravitation is $\mathrm{g}=9.81$. The minimum water height allowed in the flux computation is $h_{\min }=10^{-6}$. The minmod limiter is used for quantity reconstruction. In the current experiments, each spatial domain is discretized into 400 cells. The CFL number used in the simulations is 1.0 .

The discrete $\mathrm{L}^{1}$ absolute error

$$
E=\frac{1}{N} \sum_{i=1}^{N}\left|\mathbf{q}\left(x_{i}\right)-Q_{i}\right|
$$

quantifies numerical error, where $\mathrm{N}$ is the number of cells. The $\mathrm{L}^{1}$ absolute error is chosen, rather than the relative error, as it applies to both zero velocity and nonzero velocity cases.

With the momentum $p=h u$ and the water stage $w$ as conserved quantities, the flux calculation requires the value of velocity $u=p / h$ and the water depth $h=w-z$.

If the water depth approaches zero, then the calculation of velocity $\boldsymbol{u}$ becomes numerically unreliable and produces unphysically large velocities at wet/dry interfaces. The problems associated with these numerical instabilities due to small water depths near a wet/dry interface are alleviated by regularizing the velocity calculation as described by Roberts et al. [4]. The regularized 
velocity

$$
u=\frac{p}{h+\epsilon / h},
$$

where $\epsilon=10^{-12}$ is a regularization parameter that controls the minimal magnitude of the denominator.

In the following two subsections, numerical results are presented. Our experiments conclude that Method $\mathrm{C}$, which is a well balanced method with reconstruction on stage $w$, water height $h$, and velocity $u$, performs better than the other methods in general.

\subsection{Steady state: a lake at rest}

Suppose that we have topography of a lake, with the lake at rest and the stage has a value of $w=4.5$ as shown in Figure 2. Wet/dry interfaces occur on the left and on the right 'ends' of the lake. In addition, wet/dry interfaces are also found in the middle of the lake due to the existence of an island with a very steep shore.

Our experiments show that the methods which approximate the bed $z$ as a continuous function fail to be well balanced. Method A and Method B maintain well balance at wet/wet interfaces, but fail around wet/dry interfaces. The failure is significant if the wet/dry interface involves large changes in the bed as demonstrated by the island given in our test.

The reason for this failure follows. When we take the approximation of bed $z$ to be continuous (as in Method A and Method B) and the water height $h$ to be nonnegative, we may have a cell with sloping water surface at wet/dry interface as shown in Figure 3 so as to maintain the conservation of the water. This leads to a nonzero flux at the interface of that cell, which then induces a nonzero momentum and so the water moves. The motion at wet/dry interface propagates to cells next to it and so on, leading to a lake which is not at rest. 


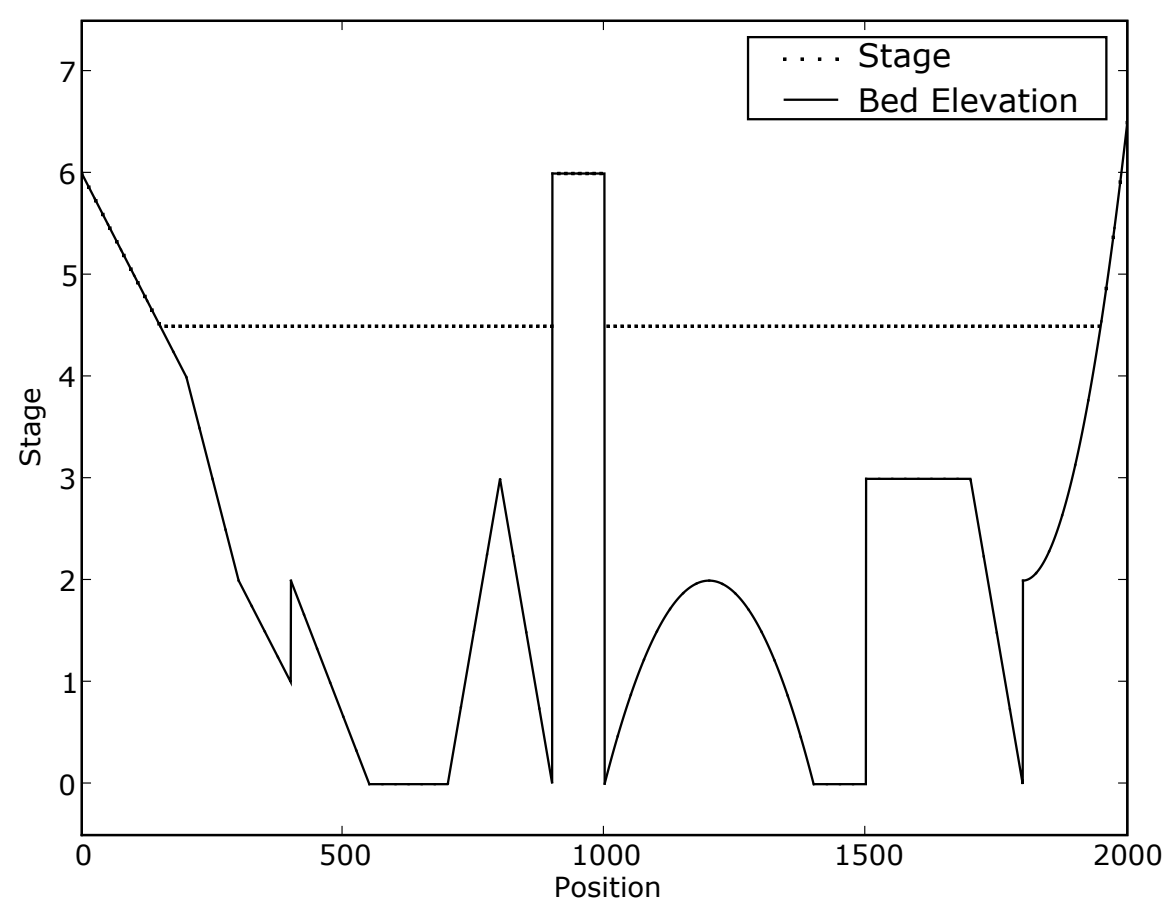

Figure 2: Cross section for topography of a lake at rest. 


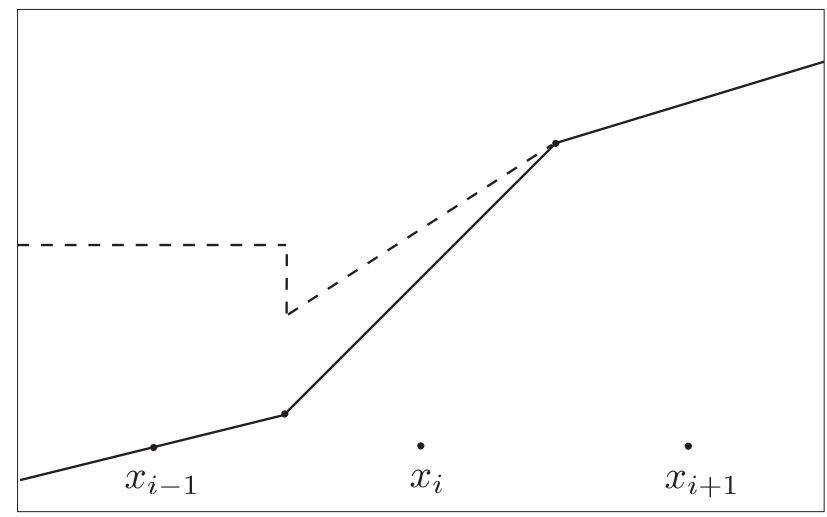

Figure 3: Piece-wise continuous linear approximation of bed $z$ at wet/dry interface: free surface (dashed line); water bed (continuous line).

Indeed the resulting velocities can be quite large. This type of numerical solution is known as a numerical storm.

In contrast, Method $\mathrm{C}$ and Method $\mathrm{D}$, which approximate the bed $z$ discontinuously, are successful in solving this steady state problem. In our simulations, the results generated by Method $\mathrm{C}$ and Method $\mathrm{D}$ at time $t=2.0$ have errors for the momentum and for the velocity on the order of $10^{-15}$ (machine accuracy).

From these results, a finite volume scheme using a well balanced flux calculation is successful to be a well balanced method if the spatial domain is all wet. However, it may fail to be a well balanced method if the spatial domain contains wet/dry interfaces. Extra care must be taken when reconstructing the quantities in this case. 


\subsection{Unsteady state: oscillation on a parabolic bed}

Consider a parabolic canal bed

$$
z=\frac{\mathrm{D}_{0}}{\mathrm{~L}^{2}} x^{2}
$$

where $D_{0}$ denotes the maximum equilibrium water depth with an equilibrium horizontal water surface length of $2 \mathrm{~L}$. Let $A$ denote the amplitude of the oscillation. We consider initial conditions in this canal, for the water velocity $u$ and stage $w$,

$$
u=0 \text { and } w=D_{0}+\frac{2 A D_{0}}{L^{2}}[x-A / 2] .
$$

The solution of this problem consists of the flat water surface moving up one side of the canal, reaching a maximum height and then falling back and then rising on the other side of the canal, and then repeating indefinitely. Therefore, the solution is an oscillation. Following Thacker [5], we find the exact (analytical) solution

$$
\begin{aligned}
& u=-A \omega \sin (\omega t), \\
& w=D_{0}+\frac{2 A D_{0}}{L^{2}} \cos (\omega t)\left[x-\frac{A}{2} \cos (\omega t)\right],
\end{aligned}
$$

where the angular frequency $\omega$ and the period $T$ of oscillation are respectively

$$
\omega=\frac{\sqrt{2 \mathrm{gD}_{0}}}{\mathrm{~L}} \text { and } \mathrm{T}=\frac{2 \pi}{\omega} .
$$

In addition, the shorelines are

$$
x=A \cos (\omega t) \pm L .
$$

Note that at dry areas we have $u=0$ and $\boldsymbol{w}=\boldsymbol{z}$. 
TABLE 2: Errors in water height $h$, momentum $p$ and velocity $u$ at $t=T$; and the maximum absolute velocity $\boldsymbol{u}_{\max }$ involved at $t=T$. Method A fails.

\begin{tabular}{c|cccc}
\hline Method & $h$ error & $p$ error & $u$ error & $u_{\max }$ \\
\hline A & - & - & - & - \\
B & $1.26 \cdot 10^{-3}$ & $1.00 \cdot 10^{-2}$ & $2.60 \cdot 10^{-3}$ & 0.4 \\
C & $1.03 \cdot 10^{-2}$ & $8.08 \cdot 10^{-2}$ & $1.94 \cdot 10^{-2}$ & 0.9 \\
D & $1.88 \cdot 10^{-3}$ & $1.37 \cdot 10^{-2}$ & $2.25 \cdot 10^{-1}$ & 7.9 \\
\hline
\end{tabular}

We use this exact solution to test the properties of the different methods of reconstruction. For our numerical experiment we consider the case where $\mathrm{D}_{0}=10, \mathrm{~L}=2500$ and $A=\mathrm{L} / 2$. The spatial domain to be discretized is the interval $[-2 \mathrm{~L}, 2 \mathrm{~L}]$.

The error comparison and the maximum absolute velocities $\mathbf{u}_{\text {max }}$ obtained at time $t=T$ (where $T$ is the period of oscillation) for the various reconstruction methods are given in Table 2. We deem that Method A fails to solve this problem since it requires artificially small time steps relative to the other methods. Method B and Method C successfully solve this problem. Method B results in the smallest error (but recall that Method B fails to solve the steady state problem as discussed in Subsection 4.1). Figure 4 shows the numerical results for Method $\mathrm{C}$ at time $\mathrm{t}=\mathrm{T}$, and Figure 5 is a closer look of Figure 4 at cells around wet/dry interface on the interval $[3500,3850]$. Method D leads to unphysical velocity in some of the dry areas as Figure 6 illustrates.

\section{Conclusions}

Many parameters are involved in our numerical simulations, such as the CFL number, minimum water height allowed in the flux computation, velocity regularization, and slope limiter. We have only taken specific values for each of these parameters and used the standard minmod limiter. Future research 

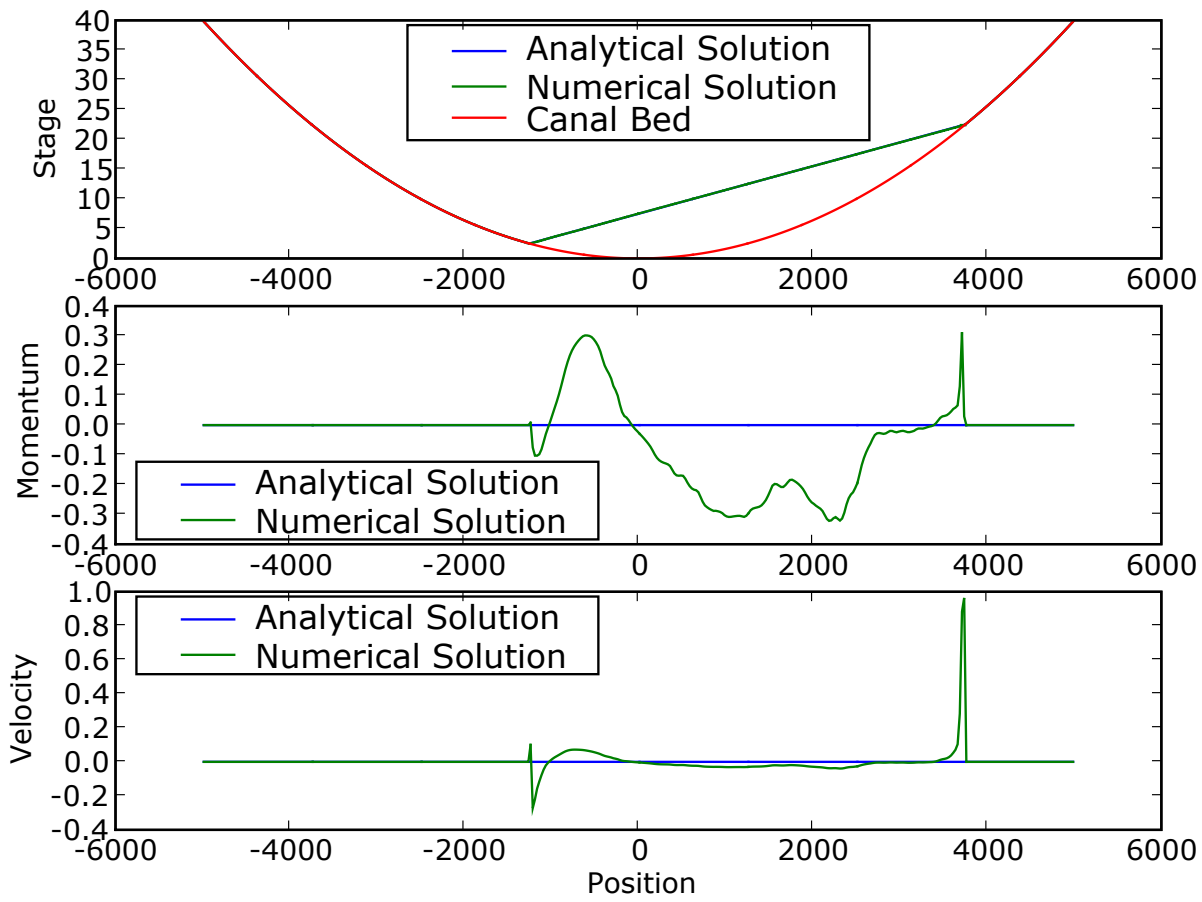

Figure 4: Simulation results at time $t=T$ using Method C. 


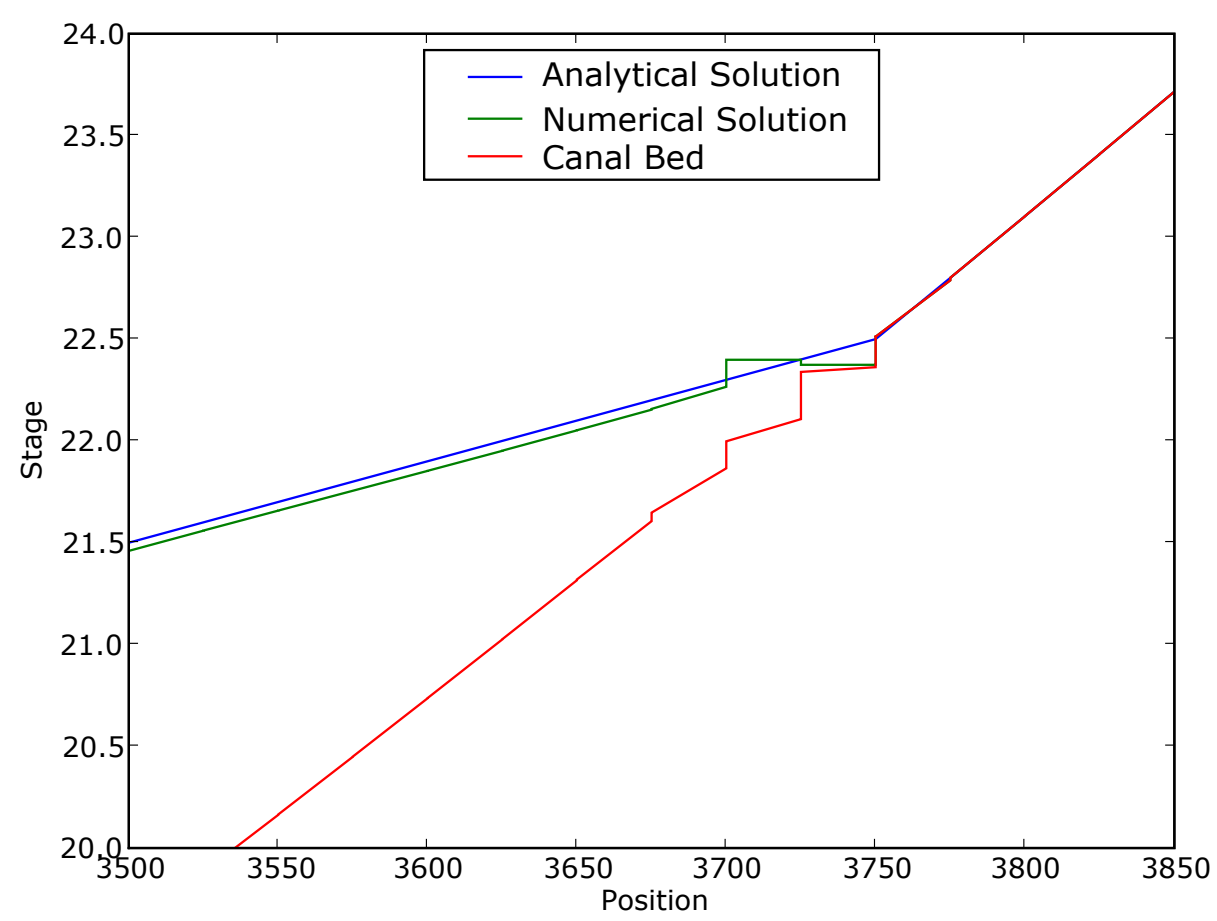

Figure 5: A closer look at cells on interval $[3500,3850]$ at time $t=T$ using Method C. The canal bed is the discretized version. 

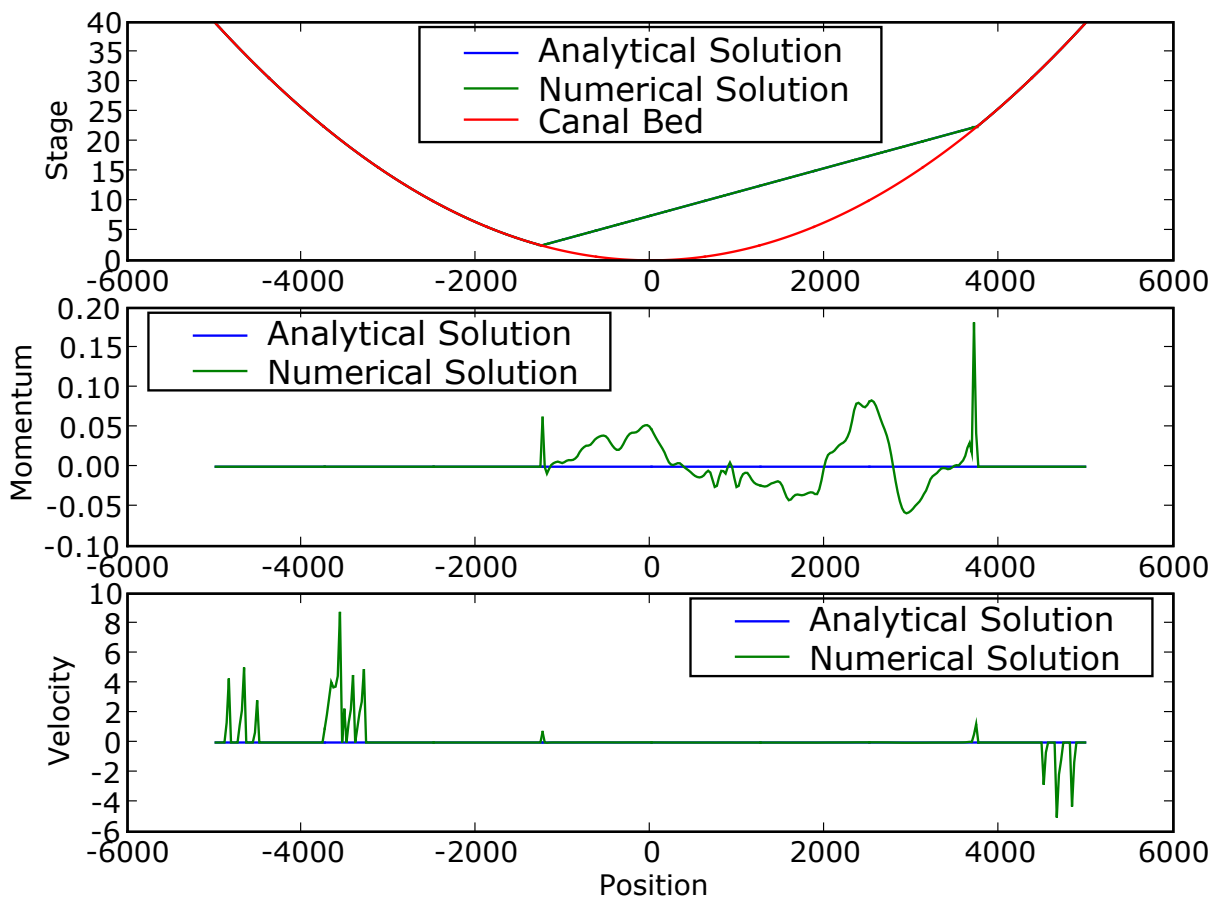

Figure 6: Simulation results at time $\mathrm{t}=\mathrm{T}$ using Method D. Unphysical velocity occurs at dry areas. 
will look for a better combination of: the values of those parameters and the slope limiter. Moreover, a combination of reconstructions able to handle both steady and unsteady state problems (reconstructions on stage, water height, and velocity) will be investigated.

Acknowledgements The work of the first author was supported by ANU $\mathrm{PhD}$ and ANU Tuition Scholarships.

\section{References}

[1] E. Audusse, F. Bouchut, M O. Bristeau, R. Klein, and B. Perthame. A fast and stable well-balanced scheme with hydrostatic reconstruction for shallow water flows. SIAM Journal on Scientific Computing, 25(6):2050-2065, 2004. doi:10.1137/S1064827503431090 C51, C52, C53

[2] A. Kurganov, S. Noelle, and G. Petrova. Semidiscrete central-upwind schemes for hyperbolic conservation laws and Hamilton-Jacobi equations. SIAM Journal on Scientific Computing, 23(3):707-740, 2001. doi:10.1137/S1064827500373413 C55

[3] S. Noelle, N. Pankratz, G. Puppo, and J R. Natvig. Well-balanced finite volume schemes of arbitrary order of accuracy for shallow water flows. Journal of Computational Physics, 213(2):474-499, 2006. doi:10.1016/j.jcp.2005.08.019 C51

[4] S. Roberts, O. Nielsen, D. Gray, and J. Sexton. ANUGA User Manual. Geoscience Australia, 2009. http://datamining. anu.edu.au/anuga C55

[5] W C. Thacker. Some exact solutions to the nonlinear shallow-water wave equations. Journal of Fluid Mechanics, 107:499-508, 1981. doi:10.1017/S0022112081001882 C59 


\section{Author addresses}

1. Sudi Mungkasi, Mathematical Sciences Institute, The Australian National University, Canberra, Australia; Department of Mathematics, Sanata Dharma University, Yogyakarta, InDONESIA. mailto:sudi.mungkasi@anu.edu.au; sudi@dosen.usd.ac.id

2. Stephen G. Roberts, Mathematical Sciences Institute, The Australian National University, Canberra, Australia. mailto:stephen.roberts@anu.edu.au 